\title{
Model-driven experimental approach reveals the complex regulatory distribution of p53 by the circadian factor Period 2
}

\author{
Tetsuya Gotoh ${ }^{a, 1}$, Jae Kyoung Kim ${ }^{b, 1,2}$, Jingjing Liu ${ }^{a, 3}$, Marian Vila-Caballer ${ }^{a, 4}$, Philip E. Stauffer ${ }^{a}$, John J. Tyson ${ }^{c}$, \\ and Carla V. Finkielstein ${ }^{\mathrm{a}, 2}$ \\ antegrated Cellular Responses Laboratory, Department of Biological Sciences, Biocomplexity Institute, Virginia Polytechnic Institute and State University \\ Blacksburg, VA 24061; ' Department of Mathematical Sciences, Korea Advanced Institute of Science and Technology, Daejeon 34131, Korea; and \\ 'Computational Cell Biology Laboratory, Department of Biological Sciences, Virginia Polytechnic Institute and State University, Blacksburg, VA 24061
}

Edited by Joseph S. Takahashi, Howard Hughes Medical Institute, University of Texas Southwestern Medical Center, Dallas, TX, and approved October 14, 2016 (received for review May 18, 2016)

The circadian clock and cell cycle networks are interlocked on the molecular level, with the core clock loop exerting a multilevel regulatory role over cell cycle components. This is particularly relevant to the circadian factor Period 2 (Per2), which modulates the stability of the tumor suppressor p53 in unstressed cells and transcriptional activity in response to genotoxic stress. Per2 binding prevents Mdm2-mediated ubiquitination of p53 and, therefore, its degradation, and oscillations in the peaks of Per2 and p53 were expected to correspond. However, our findings showed that Per2 and p53 rhythms were significantly out-of-phase relative to each other in cell lysates and in purified cytoplasmic fractions. These seemingly conflicting experimental data motivated the use of a combined theoretical and experimental approach focusing on the role played by Per2 in dictating the phase of p53 oscillations. Systematic modeling of all possible regulatory scenarios predicted that the observed phase relationship between Per2 and p53 could be simulated if (i) p53 was more stable in the nucleus than in the cytoplasm, (ii) Per2 associates to various ubiquitinated forms of p53, and (iii) Per2 mediated p53 nuclear import. These predictions were supported by a sevenfold increase in p53's half-life in the nucleus and by in vitro binding of Per2 to the various ubiquitinated forms of p53. Last, p53's nuclear shuttling was significantly favored by ectopic expression of Per2 and reduced because of Per2 down-regulation. Our combined theoretical/mathematical approach reveals how clock regulatory nodes can be inferred from oscillating time course data.

tumor suppressor $\mid$ circadian rhythms $\mid$ p53 | mathematical modeling | Period 2

7 he he circadian clock is an autonomous molecular mechanism that controls biochemical, physiological, and behavioral processes with a periodicity of $24 \mathrm{~h}$ in living organisms and can be entrained by environmental cues (for review, see ref. 1). The clock is sustained by a coordinated interplay of positive and negative transcriptional-translational feedback loops driven by circadian factors, a core group of proteins that either possess intrinsic transcriptional activity or modulate gene expression (1). Disruption of the circadian clock by either desynchronizing the light/dark cycle entrainment, altering sleep/wake/feeding behavior, or manipulating the expression of core circadian genes result in an increased risk of development, progression, and exacerbation of a host of disease states, including proliferative disorders (for review, see refs. 2 and 3 ).

Circadian factor protein Period 2 (Per2) is a large and multifaceted protein with an incompletely defined domain architecture, whose functional relevance extends beyond its role as a circadian regulator (for review, see ref. 4). Loss of Per2 function predisposes genetically engineered mice to spontaneous lymphomagenesis, increases susceptibility to radiation, and influences cell growth, division, and cell death in various models of human cultured cells (for review, see ref. 5). Whereas the above findings are consistent with a role for PER2 in suppressing a cancer-prone phenotype, a note of caution is in order, as inconsistencies among in vitro and in vivo studies have been recently reported $(6,7)$.

At present, our understanding of the signaling events linking Per2 to arrest of proliferation and to cell death are just beginning to emerge. We now know that, in unstressed cells, Per2 transcriptionally modulates TP53 and directly associates with the C-terminus region of the p53 transcription factor (p53), which prevents murine double minute-2 (Mdm2)-mediated ubiquitination of p53 and further degradation by the proteasome pathway (8). As a result, low levels of p53 remain present at all times in the cell allowing for a "preconditioning" state to exist. This primes the cell for a rapid response to genotoxic stress, which is further sustained by a Per2induced p53 transcriptional loop $(8,9)$. As Per2 blocks p53's oligomeric domain, which is needed for the formation of tetrameric transcriptionally active p53, p53's downstream response to genotoxic stress not only depends on its availability in the system but also on its dissociation from Per2 in the nucleus (9). Furthermore, it is the spatiotemporal distribution of Per2:p53 that, ultimately,

\section{Significance}

Cells sense changes in environmental conditions and translate them into physiological responses that are mediated by a plethora of molecular intermediaries that converge at critical nodes. This is particularly relevant to multiple levels of biological organization in which the circadian clock and cell division are interlocked. Unexpectedly, the circadian rhythms of two critical components of the interlocked system, Period 2 (Per2) and p53, were in antiphase. This finding was analyzed using a systematic modeling approach, and predictions of the model were validated by experimental studies that revealed a distinct and complex scenario. Accordingly, we show that the spatiotemporal organization of Per2:p53 interactions and the nature of their chemical modifications are critical for their time-dependent subcellular redistribution and potential biological functions.

Author contributions: T.G., J.K.K., and C.V.F. designed research: T.G., J.K.K., J.L., M.V.-C, and P.E.S. performed research; C.V.F. supervised and coordinated all investigators for the project; T.G., J.K.K., J.J.T., and C.V.F. analyzed data; and J.K.K. and C.V.F. wrote the paper. The authors declare no conflict of interest.

This article is a PNAS Direct Submission.

Freely available online through the PNAS open access option.

${ }^{1}$ T.G. and J.K.K. contributed equally to this work.

${ }^{2}$ To whom correspondence may be addressed. Email: finkielc@vt.edu or jaekkim@kaist.ac.kr. ${ }^{3}$ Present address: Molecular and Cellular Oncogenesis, The Winstar Institute, Philadelphia, PA 19104

${ }^{4}$ Present address: Department of Biomedical Sciences, University CEU Cardenal Herrera, Valencia 46113, Spain.

This article contains supporting information online at www.pnas.org/lookup/suppl/doi:10 1073/pnas.1607984113/-/DCSupplemental. 
provides the unifying framework with which to understand the link between the two components of our initial model. These findings place Per2 as a component of the checkpoint response by intersecting the $\mathrm{p} 53$ node, thus providing a mechanistic account for some of the Per2-associated growth inhibitory and proliferative phenotypes observed in cultured cells and in knockout animals, respectively, where endogenous levels of Per2 were experimentally modulated (10-12).

Using an approach that combines mathematical modeling of the protein interaction network with targeted experiments, we established the asymmetric distribution of Per2:p53 in the cell and its impact on the time-dependent regulatory mechanism that modulates p53's rhythmic behavior, stability, and cellular distribution. Our models and data further revealed the relevance of posttranslational modification events that take place in separate cellular compartments and that contribute to the time-dependent phase shift in the accumulation of p53 proteins.

\section{Results}

Rhythmic Accumulation of p53 and Per2 Are Noticeably Out-of-Phase. The observation that Per2 binding to p53 favors its stability by preventing Mdm2-mediated polyubiquitination of $\mathrm{p} 53$ prompted us to investigate whether p53 oscillation closely follows Per2 rhythms (Fig. 1A). Accordingly, extracts from circadian synchronized human colorectal carcinoma-116 (HCT116) cells were monitored for their level of expression of Per2, chryptochrome 1 (Cry1), $\mathrm{Mdm} 2$, and p53 proteins in a time-dependent fashion (Fig. 1A, Upper). As expected, Cry1 and Per2 undergo similar oscillations, albeit with a short delay, with a periodicity of about $24 \mathrm{~h}$, following their mRNA by a few hours (Fig. $1 B$ and Fig. S1 $A$ ). Importantly, p53 was comparatively low at $0-4 \mathrm{~h}$ post serum shock, rose to a maximum at 12-16 h, and slowly decreased afterward (Fig. 1A). Thus, the peaks of Per 2 and p53 expression in total cell extracts (named Per $2_{\text {te }}$ and $\mathrm{p} 53_{\text {te }}$ hereafter) is shifted by $\sim 8 \mathrm{~h}$ (out-ofphase), and the amplitude of the p53 peak is reduced compared with that of Per2 (Fig. 1A, Lower). Moreover, the out-of-phase relationship between Per2 and p53 remains a prevalent feature among cell-based systems and in response to different circadian stimuli as is the case, for example, in HeLa cells and with treatments such as dexamethasone (Fig. S1 $B$ and $C$ ).

Next, we investigated whether p53 rhythms stem from the rhythmic transcription of TP53 or, instead, its negative regulator, the E3 ubiquitin protein ligase Mdm2. In contrast to the strong rhythmically expressed changes observed for PER2 mRNA (Fig. 1B), quantitative RT-PCRs (qRT-PCRs) exposed very weak rhythmic levels of the TP53 transcript. This was not unexpected as TP53's transcript half-life is $\sim 9 \mathrm{~h}$, which is much greater than that of Per2 $(\sim 0.7 \mathrm{~h})(13-15)$. Therefore, as the half-life of p53 protein is much shorter $(\sim 2 \mathrm{~h})$ than its mRNA (8), circadian modulation of p53 is likely to be more effective at the protein level than at the gene expression level. Furthermore, taking into account that the peak of the p53 protein precedes that of its transcript, it is unlikely that the rhythms in protein level result from rhythms in gene expression. Last, we examined whether antiphase expression of Mdm2 could drive the rhythmic accumulation of p53. Accordingly, detection of Mdm2 levels was by immunoblotting, and quantitative studies were carried out using JTK CYCLE, a nonparametric algorithm testing rhythmicity (Fig. $1 A$ and Table S1) (16). Whereas our results confirm the absence of rhythmic levels of Mdm2, a note of caution should be raised, as other scenarios involving Mdm2 cannot be excluded (e.g., posttranslational events, subcellular localization) that might contribute to the observed pattern of p53 expression.

These findings further motivate the search for the formation of the Per2:p53 complex in extracts from synchronized cells. Accordingly, we examined how Per2 and p53's phase relationship impact the formation of the Per2:p53 complex over time. Extracts from circadian synchronized HCT116 cells were analyzed

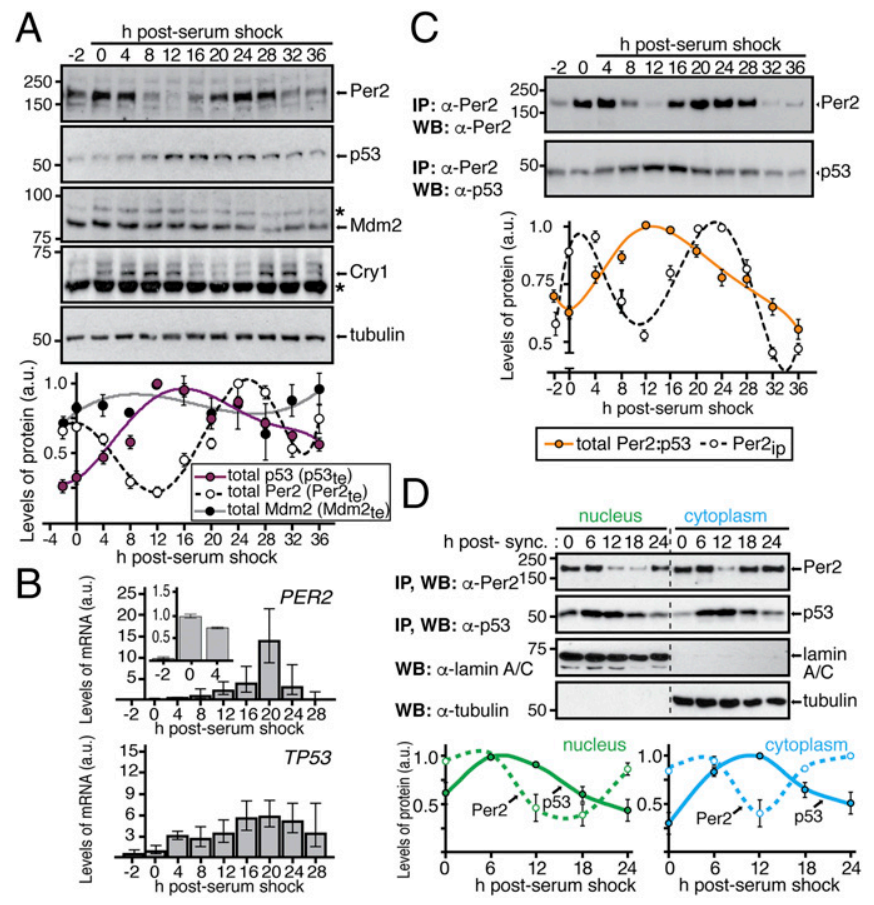

Fig. 1. Distinct p53 and Per2 phases characterize their time-dependent subcellular distribution. ( $A$, Upper) Extracts from circadian synchronized HCT116 cells were analyzed for the expression of endogenous Per2, p53, Mdm2, and Cry1 by immunoblotting. Asterisks indicate nonspecific bands. (Lower) Bands were quantified using ImageJ, and values were normalized to tubulin levels. Data are in arbitrary units (a.u.). (B) Samples from $A$ were processed for qRT-PCR as described in SI Materials and Methods. Data for PER2 and TP53 gene expression are shown as the mean \pm SEM from three independent experiments performed in triplicate. Bar graphs are fold increase normalized to the level of expression at $t=0 \mathrm{~h}$. Inset indicates level of Per2 expression within the first $4 \mathrm{~h}$. (C) HCT116 extracts from various times postcircadian synchronization ( $t=0-36 \mathrm{~h}$ ) were immunoprecipitated using $\alpha$-Per2. Bound proteins were identified by immunoblotting and quantified as described in $A$. Relative amounts of Per2 and total Per2:p53 complex were plotted in arbitrary units relative to $t=0$. (D) Nuclear and cytoplasmic fractions from circadian synchronized HCT116 cells were enriched for endogenous Per 2 and $\mathrm{p} 53$ by immunoprecipitation and blotted using $\alpha$-Per 2 or -p53 antibodies, respectively. Tubulin and lamin A/C were used as controls. Bands were quantified and plotted as in $A$. In $A, C$, and $D$, immunoblot data were originated from a single experiment that was repeated three times with similar results. Error bars represent mean \pm SEM.

for the presence of total Per2 levels and endogenous Per2:p53 and Per2:Cry1 complexes by immunoprecipitation (Fig. $1 C$ and Fig. S1 $D$ and $E$ ). The Per2:p53 complex showed a peak at 8-16 h after synchronization (Fig. $1 C$, Lower), which is in clear contrast with the level of total Per2 detected in cell extracts (Fig. $1 C$, Upper) and is shifted from that of Per2:Cry1 (Fig. S1E), which is depicted in the quantitative profile. Because Per2 stabilizes p53 (8), the lack of correspondence between the Per2 peak and both the p53 (Fig. $1 A$ ) and Per2:p53 (Fig. $1 C$ ) peaks in total extracts implies the existence of an additional level of regulation (i.e., posttranslational) that needs to be factored into our initial model (9).

We then investigated whether the Per2 and p53 phase shifts identified in total extracts resulted from a complex redistribution of these proteins in different cellular compartments. The rationale behind this hypothesis is based on findings that Per2 shuttles between compartments following an oscillatory pattern that leads to an uneven distribution of the protein over time (17) and that p53 undergoes nuclear-cytoplasmic shuttling in a process that is both rapid and energy dependent $(18,19)$. We monitored Per2 and p53 protein 
levels in nuclear and cytoplasmic fractions purified from circadian synchronized HCT116 cells (Fig. 1D). Unexpectedly, whereas the rhythms of Per2 and p53 were in-phase in the nucleus, both proteins exhibited an out-of-phase relationship in the cytoplasm (Fig. $1 D$ ). In context with our previous findings (8), these results established that the phase relationship between p53 and Per2 observed in total extracts overlooks the role of compartmentalization.

Next, we wanted to evaluate whether the phase relationships for Per2 and p53 in either compartment were maintained even when the circadian clock was perturbed (Fig. S2). Consequently, HCT116 cells were circadian synchronized by serum shock followed by addition of PF670462 (named PF670 hereafter), a potent dual pharmacological inhibitor of casein kinase $1 \varepsilon / \delta(\mathrm{CK} 1 \varepsilon / \delta)$ that causes lengthening of clock oscillations by stabilizing Per2 (20). Lysates and subcellular fractions were quantitatively analyzed to evaluate the level of Per2, p53, Mdm2, and CK1 1 expression (Fig. $\mathrm{S} 2 A$ and $B$ ). As expected, addition of PF670 stabilized Per2 in both compartments (Fig. S2 $A$ and $B$, Upper) and delayed the circadian phase (Fig. S2C). This is in agreement with the established role of CK1 $1 / \delta$-mediated phosphorylation of Per2 for its turnover and in sustaining the negative loop (21). Remarkably, and unlike Mdm2 and CK1 proteins (Fig. S2 $A$ and $B$, Lower), the rhythms of Per2 and p53 were both delayed in PF670-treated samples in a manner that mimics the phase relationship observed in the absence of PF670 treatment (Fig. S2 $A$ and $B$, Upper). Last, the activity of $\mathrm{CK} 1 \varepsilon / \delta$ relevant to Per2's stability and Per:Cry heterodimerization is not a contributing factor, at least under unstressed conditions, in regulating phase relationship between Per2 and p53. Instead, Per2 acts as the chief regulator (Fig. S2C).

Mathematical Modeling Predicts Underlying Interactions Between Per2 and p53. The inherent complexity of the interplay between Per2 and p53 begs for a combined theoretical and experimental approach to aid in the interpretation of experimental data, to generate testable predictions, and to develop a unified understanding of the phenomena $(22,23)$. Accordingly, the phase relationship between Per2 and p53 (Fig. 1) was analyzed in the context of all possible scenarios in which rhythmic Per2 could influence production, nucleus shuttling (entry/exit), and degradation of p53 (Fig. $2 A$ ). For each scenario, regulation types were randomly selected among positive $(+)$, null $(0)$, or negative $(-)$ interactions during the model fitting process (Fig. $1 D$ and SI Materials and Methods). As a result, we found $10^{3}$ predicted models that successfully simulated the observed phase relationship between p53 and Per2. Interestingly, although the regulation types were randomly chosen, Per2-mediated entry of p53 to the nucleus was strongly skewed in the positive direction among all models, indicating that this event is essential to accurately simulate the rhythms of p53 (Fig. 2B). An additional unifying property among the $10^{3}$ models predicted that the stability of nuclear p53 should be greater than that of the protein in the cytoplasmic compartment to successfully simulate p53 rhythms (Fig. 2C).

Based on these initial two findings (Fig. $2 B$ and $C$ ), we developed a more detailed and realistic mathematical model (Fig. $2 D$, Tables S2 and S3, and SI Materials and Methods). First, we modified the Kim and Forger's model used to represent the dynamics of the transcriptional negative arm generated by Per2 $(24,25)$. Second, we considered the ubiquitination status of p53 as it modulates p53 degradation and shuttling (26). Specifically, the dual role of monoubiquitination in p53 shuttling as a mode to impede nuclear import while facilitating p53 nuclear exit was addressed (18). In addition, the relevance of polyubiquitination in $\mathrm{p} 53$, which is associated with its rapid turnover (18), is reflected in the model by rapid clearance of $\mathrm{p} 53$ in the cytoplasm compared with the nucleus (Fig. 2C). Third, the model incorporates the interaction of Per2 with p53, which modulates p53 stability and function $(8,9)$. Accordingly, when p53 is prebound to Per2, both monoubiquitination and polyubiquitination of $\mathrm{p} 53$ are inhibited (8), thereby promoting $\mathrm{p} 53$ nuclear translocation (Fig. $2 B$ ) while blocking its proteasomal degradation.

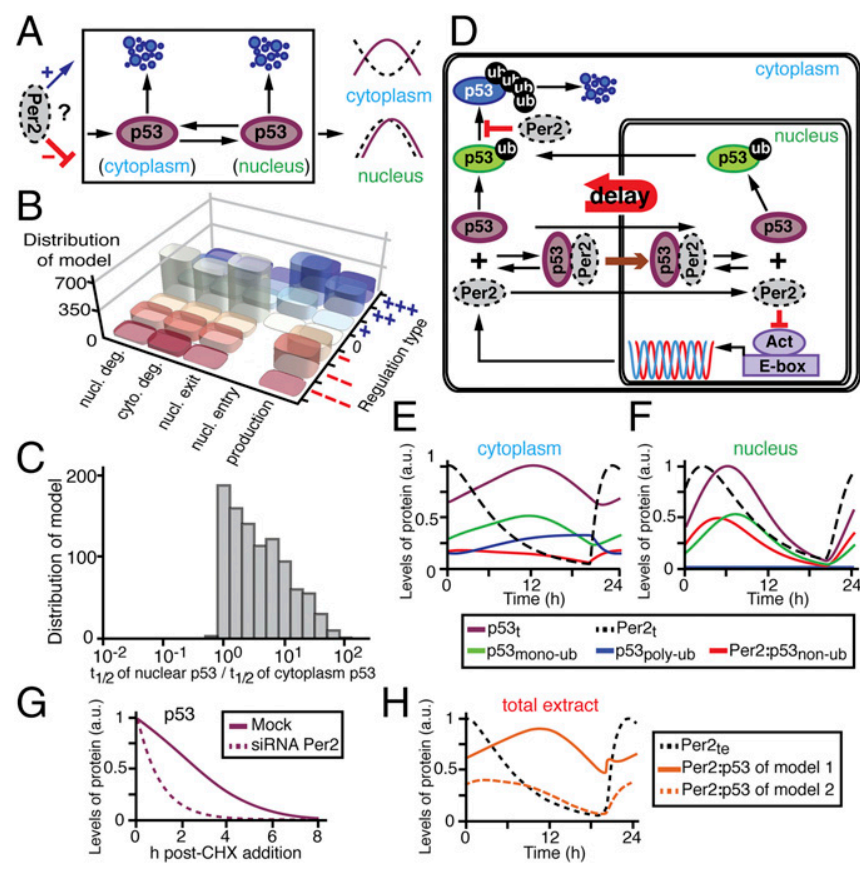

Fig. 2. Mathematical modeling predicts unexpected mechanisms for Per2 mediated regulation of $\mathrm{p} 53$ phases. $(A)$ Schematic representation of potential regulatory nodes for which Per2 can influence p53 phases. For each regulation, its type is randomly selected among positive $(+) /$ null (0)/negative $(-)$ throughout the process of fitting the model to the observed p53 and Per2 rhythms (see SI Materials and Methods for details). (B) Distribution of the $10^{3}$ successful models according to their regulation type for $\mathrm{p} 53$. The strengths of regulation types are represented as the number of + and - (see SI Materials and Methods for details) (C) Distribution of the $10^{3}$ successful models according to the relative half-life of nuclear vs. cytoplasmic p53. (D) Simplified representation of a comprehensive model for Per2 and p53 interaction based on the two essential properties predicted in $B$ and $C$. $(E$ and $F$ ) Simulated time course levels of Per2 and p53 species in both cytoplasm $(E)$ and nuclear $(F)$ compartments (see Fig. S3 for total extracts). $(G)$ Prediction of p53 half-life in the presence or absence of Per2 in the system. $(H)$ Phase predictions for the Per2:p53 complex differ depending on whether Per2 binding occurs regardless of p53 ubiquitination status (model 1 ) or is only associated to nonubiquitinated p53 (model 2).

Next, we confirmed that the simulated time courses of Per2 and p53 (Fig. $2 E$ and $F$, and Fig. S3) matched the experimental data equally well (Fig. $1 A$ and $D$ ). In particular, the model predicted that the differences in phases observed between the p53 and Per2 peaks in the cytoplasm (Fig. $1 D$ ) were largely due to Per2mediated shuttling of p53 into the nucleus, where p53 remains stable (Fig. $2 B$ and $D$ ). In this scenario, when Per2 levels are high (Fig. $2 E$ and $F ;$ Per 2 t, dashed black line), the cytoplasmic-to-nuclear translocation of nonubiquitinated $\mathrm{p} 53$ is promoted [Fig. $2 D$, brown arrow, and Fig. $2 F$; Per2:nonubiquitinated p53 (p53 nonub), red solid line]. Once in the nucleus, the Per2:p53 nonub complex dissociates allowing for free p53 to be monoubiquitinated [Fig. $2 F$; monoubiquitinated p53 (p53 monoub), green solid line], stabilized, and ultimately exported to the cytoplasm (27). Consequently, export of p53 monoub results in a peak delay of p53 levels in the cytoplasm (Fig. $2 D$ and $E$, green solid line). A corollary to that prediction is that p53 half-life should be shorter in a $P E R 2^{-/-}$background (Fig. $2 G$ ) as demonstrated in ref. 8. Last, the model predicts that the phase of the Per2:p53 complex strongly depends on the ubiquitination status of p53 (nonubiquitinated, monoubiquitinated, or polyubiquitinated) that binds to Per2 and forms the complex (Fig. $2 H$ ). Accordingly, if binding of Per 2 only occurs to nonubiquitinated p53, the phase of the Per2:p53 complex is predicted to be in-phase with that of total Per2 (Fig. $2 H$, orange dashed line) in clear contrast to the results 
shown in Fig. 1C. Alternatively, if Per2 binding occurs regardless of the ubiquitination status of $\mathrm{p} 53$, rhythms between the complex and total Per2 species are predicted to be antiphase (Fig. $2 H$, orange solid line) and in agreement with the experimental results shown in Fig. 1C. Overall, these predictions provide an initial framework for understanding what, a priori, was an unexpected observation (Fig. $1 A)$. Therefore, we set out to test these specific predictions of the model experimentally.

The Localization of p53 Influences the Kinetics of Its Degradation. The model predicts that three essential properties simulate the correct rhythms of p53 (Fig. $2 B, C$, and $H$ ). To test these predictions, initial experiments were devoted to evaluating whether p53 half-life differed in either the cytoplasmic or the nuclear compartment using cycloheximide (CHX). Whereas treatment of cells with CHX (an inhibitor of protein biosynthesis) can have a biasing effect on degradation rates of proteins in vitro and under normal growth conditions, and in different subcellular locations (28), this approach has been successfully used to compare the half-life of other core clock proteins in nuclear and cytoplasmic compartments (29). Therefore, HCT116 cells were treated with $\mathrm{CHX}(t=0)$ and harvested at different times after treatment (Fig. 3; $t=1-4 \mathrm{~h}$ ). The level of p53 was evaluated in both nuclear and cytoplasmic fractions as well as in whole-cell lysates by immunoblotting (Fig. 3 and Fig. S4). In agreement with the model's prediction (Fig. 2C), the half-life of endogenous p53 in the nucleus was found to be considerably longer than that of p53 in the cytoplasmic compartment (Fig. 3B, Upper; $t_{1 / 2 \mathrm{n}} / t_{1 / 2 \mathrm{c}} \sim 7$ times) where p53 is normally polyubiquitinated and targeted for degradation (30). Unlike p53, Per2 half-life remained unchanged in each compartment for the time course analyzed (Fig. $3 B$, Lower; $t_{1 / 2 \mathrm{n}} / t_{1 / 2 \mathrm{c}} \sim 1$ ). These results further motivate the need to understand how Per2 shuttling influences the dynamic distribution of p53.

Qualitative Assessment of Per2 Association to the Various Ubiquitination Forms of p53. We previously showed that Per2 binding to p53 prevents subsequent p53 ubiquitination by $\mathrm{Mdm} 2$ (8). Whereas this interaction provides a means for enhancing p53's stability and availability in the cell and for a rapid response to genotoxic stress (9), our model predicts that other ubiquitinated forms of p53 should be amenable to Per2 binding (Fig. $2 H$ ). To test this possibility in vitro, we performed a sequential ubiquitination and binding assay and monitored the presence of Per2 bound to the various ubiquitinated p53 forms (Fig. 4 and Fig. S5A). In vitro-transcribed and -translated $m y c$-p53 was incubated with FLAG-Mdm2 in the

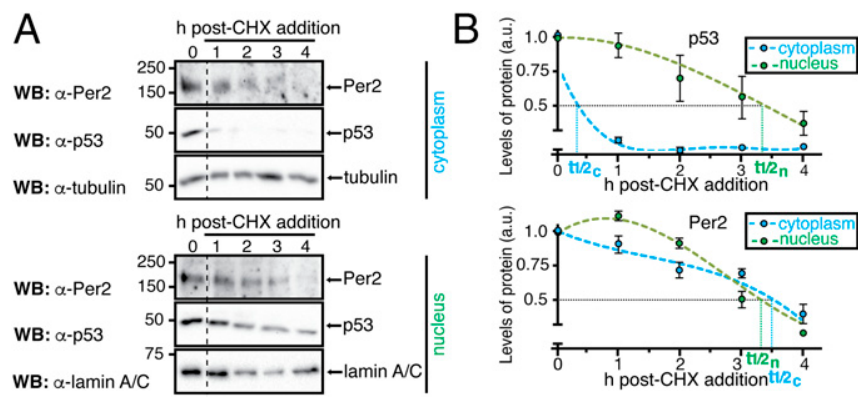

Fig. 3. The half-life of $p 53$, but not Per2, differs in nuclear and cytoplasmic compartments. $(A)$ Samples were obtained from HCT116 cells in the absence $(t=0)$ or presence of $\mathrm{CHX}$ as indicated in SI Materials and Methods section. Extracts from cytoplasmic and nuclear fractions were analyzed by immunoblotting using $\alpha$-Per2 and -p53 antibodies. Tubulin and lamin A/C were used as controls. ( $B$ ) Protein levels were quantified as described in SI Materials and Methods section, values were normalized to tubulin or lamin A/C levels, and p53 and Per2 half-life $\left(t_{1 / 2}\right)$ calculated using Excel. Error bars represent mean \pm SEM of three independent experiments.

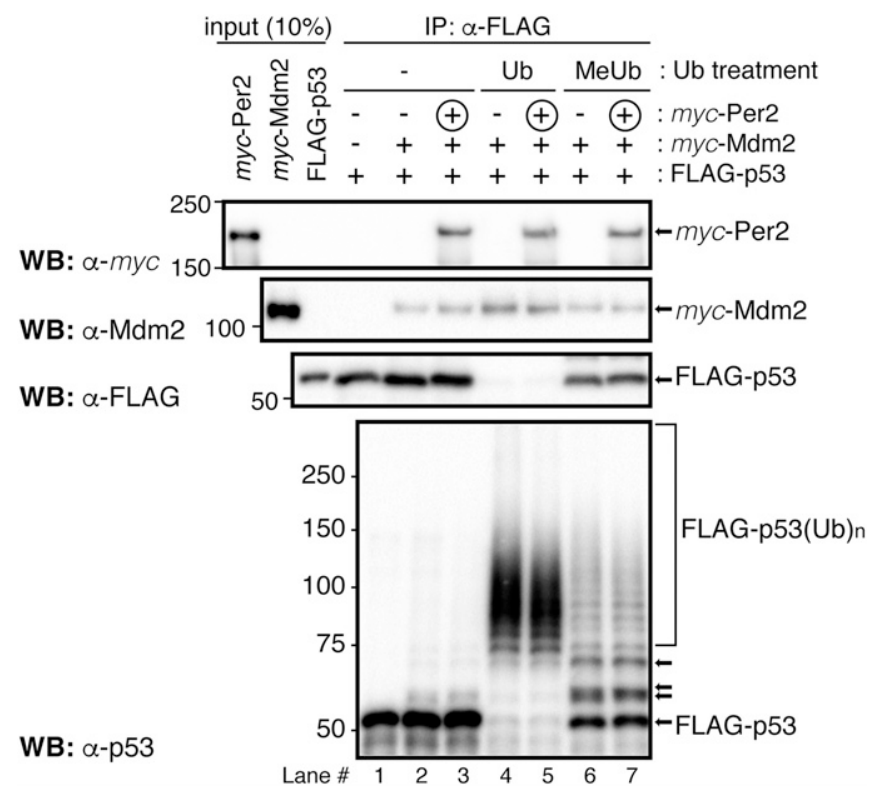

Fig. 4. Per2 associates to ubiquitinated forms of $\mathrm{p} 53$. In vitro-transcribed and -translated FLAG-p53 was preincubated (+) with myc-Mdm2 and the complex added to a reaction mixture containing (+) or not $(-)$ the ubiquitin substrate (ubiquitin, Ub, or methyl-ubiquitin, MeUb) as indicated in SI Materials and Methods and Fig. S5A. Circled "+" indicates myc-Per2 was added after complexes were formed and washed. Bound proteins were detected by immunoblotting. The FLAG-p53(Ub) $)_{n}$ forms were detected using $\alpha-p 53$ antibody (Lower). Arrows indicate examples of monoubiquitinated forms of $\mathrm{p} 53$.

presence (or absence) of a ubiquitin mixture and MG132, a proteasome inhibitor. Polyubiquitinated forms of p53 were purified and tested for Per2 binding by immunoprecipitation and blotting (Fig. 4 and Fig. S5A). As shown in Fig. 4 and Fig. S5B, p53 is efficiently polyubiquitinated by Mdm2 (lanes 2 and 3 vs. 4 and 5, Lower) and, despite this chemical modification, Per 2 binding still occurs (lanes 3 vs. 5). When methyl-ubiquitin, which prevents elongation but not initiation of ubiquitin chains, was supplemented to the mixture instead, only monoubiquitinated p53 was generated. In accordance with our prediction, binding of Per2 was detected (Fig. 4 and Fig. $\mathrm{S} 5 B)$. Overall, these results, and those presented in Figs. $1 C$ and $2 H$, support a model in which Per2 is capable of associating with p53 regardless of p53's ubiquitination status and, thus, contributes to the pool of Per2:p53 complex whose distribution is antiphase to that of Per2. Still, much remains unknown regarding the underlying biochemistry that surrounds the association between Per2 and p53 as p53's ubiquitination might influence, at multiple levels, the affinity of p53 for Per2 and/or that of its ternary partner Mdm2.

Expression of Per2 Favors p53 Nuclear Translocation. An essential property predicted by our model is that, to simulate the correct phase of p53 rhythm, Per2 should positively influence p53 nuclear translocation (Fig. $2 B$ ). Further experiments were done to confirm this prediction by evaluating the influence of Per2 expression levels on p53 trafficking. Initially, HCT116 cells were transfected to express trace levels of myc-Per2, low enough to keep total levels of Per2 relatively constant in the cell and avoiding potential overexpression artifacts (Fig. 5A, Upper). In fact, detection of the tagged form of Per2 was marginal to the point that only the addition of six tags in the construct allowed for its identification (Fig. 5A, Middle, and Fig. S6 $A$ ). To better capture p53 distribution, experiments were performed in the presence of both MG132, a proteasome inhibitor, to block p53 degradation, and/or leptomycin B (LMB), which, by inhibiting the nuclear export factor CRM1, prevents p53 nuclear exit. As 

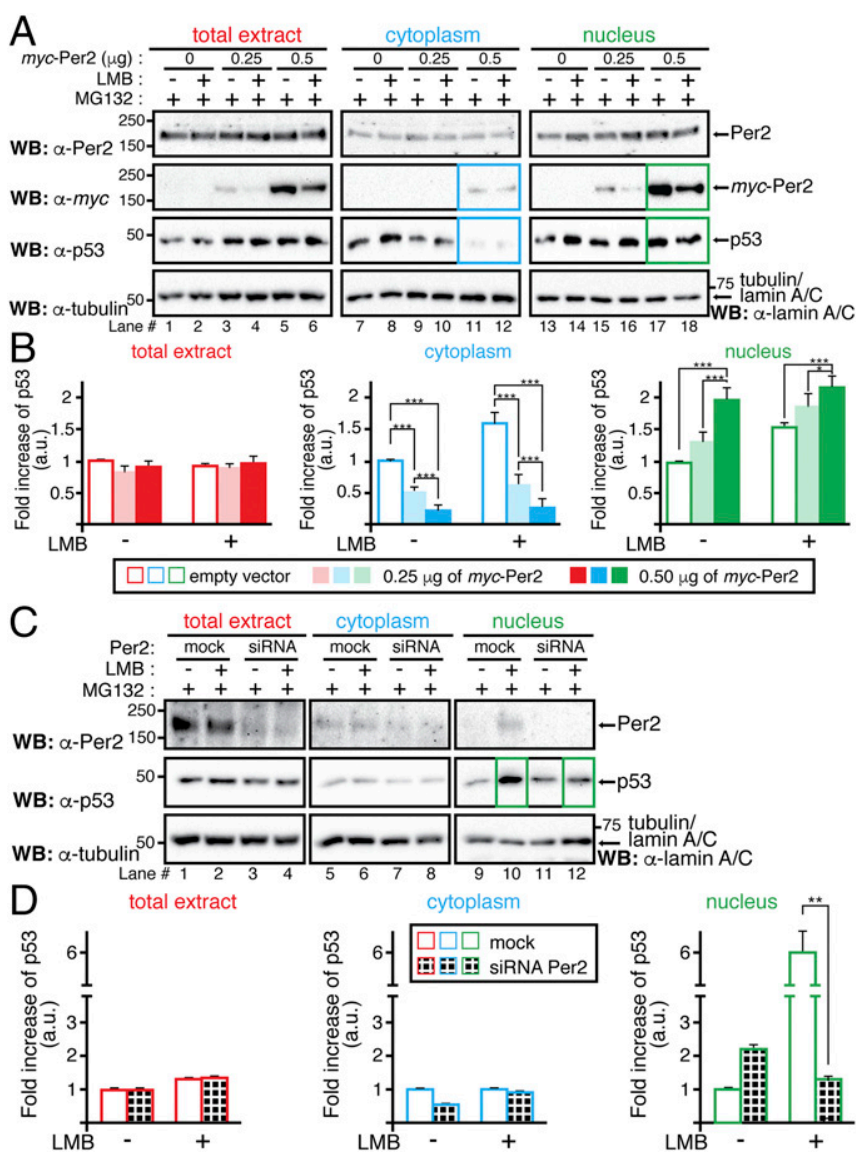

Fig. 5. Per2 enhances p53 shuttling to the nucleus. (A) HCT116 cells were transfected with either myc-Per2 $(0.25$ or $0.5 \mu \mathrm{g})$ or empty vector $(0)$ for $24 \mathrm{~h}$ before the addition of MG132 and/or leptomycin B (LMB). Lysates were subjected to subcellular fractionation, and total extract (Left), cytoplasmic (Middle), and nuclear (Right) fractions were analyzed for the presence of Per2, myc-Per2, and endogenous p53 by immunoblotting. Tubulin and lamin A/C were used as controls. (B) Endogenous p53 was quantified using Image Lab software/Gel Doc $\mathrm{XR}+$ system and values normalized to tubulin or lamin AVC levels. (C) HCT116 cells were transfected with either scrambled siRNA (mock) or Per2 siRNA (siRNA) for $48 \mathrm{~h}$ before the addition of MG132 and/or LMB. Endogenous proteins were detected as indicated in $A$ and quantified as in $B$. In $B$ and $D$, data are represented as fold increase of p53 (in arbitrary units) compared with similar treatment in either vector-transfected or mock samples, respectively. Values are the mean \pm SEM from three independent experiments. Statistical significance was determined by $t$ test. ${ }^{*} P \leq 0.05 ; * * P \leq 0.005, * * * P<0.001$.

shown in Fig. $5 A$, augmented expression of $m y c$-Per2 resulted in a concomitant decrease in p53 levels in the cytosol (lanes 5 and 6 vs. 11 and 12) and increased detection of both components in the nucleus (lanes 11 and 12 vs. 17 and 18), an observation that was further supported by quantitative analysis and statistical inference (Fig. 5B). Indeed, the converse experiment, where endogenous Per2 levels were down-regulated by small interfering RNA (siRNA) transfection (Fig. $5 C$ and Fig. S6B), resulted in a sharp decrease of nuclear p53 accumulation (Fig. $5 C$, lanes 10 vs. 12 and Fig. 5D). Although existing shuttling mechanisms for p53 are in place in the cell (26), our results support a model in which partial translocation of p53 and its time-of-day cellular distribution are mediated by the availability and binding capability of Per2.

\section{Discussion}

Today, evidence points toward circadian factors controlling numerous processes that impact cell cycle transitions, growth, and death (for review, see ref. 31). However, we lack a unifying view about how those interactions operate under normal progression and how they adjust to stress conditions. To begin addressing this shortcoming, we focus on circadian components, specifically Per2, which play critical roles in controlling proliferation by interacting with cellular factors at key regulatory nodes. Accordingly, our previous findings placed Per2 at the core of the checkpoint response by directly interacting with p53, controlling p53's stability in unstressed conditions and its transcriptional activity in response to genotoxic stimuli $(8,9)$; however, little is known about how Per2 and p53's spatiotemporal distributions help to segregate signals in the cell.

Our approach is simple in concept as it combines theoretical and experimental work and is motivated by an existing conundrum: how can discordant Per2 and p53 phases coexist in a model in which Per2 enhances p53's stability (Fig. 1 and ref. 8)? To tackle this problem, we used a systematic investigation of models from which we could infer the interactions between Per2 and p53 using their time course data (Fig. 1 and refs. 22, 23, and 32). Three essential molecular mechanisms were predicted (Fig. 2 and Fig. S3) and validated experimentally: $(i)$ p53 half-life is greater in the nucleus (Fig. 3 and Fig. S4), (ii) Per2 associates to various ubiquitinated forms of p53 (Fig. 4 and Fig. S5), and (iii) Per2 promotes p53's nuclear entry (Fig. 5 and Fig. S6). Accordingly, the proposed model implies that the phase difference between Per2 and p53 in the cytoplasm may result from Per2:p53 binding and shuttling between compartments where posttranslational modifications in p53 take place (Fig. 6).

Protein translocation dynamics and posttranslational events are recurrent themes when analyzing the importance of oscillations for modulating cell cycle transitions and for the generation of circadian rhythms. We know now that what makes the regulation of $\mathrm{p} 53$ by Per2 distinctive from others, besides Per2's role as a p53 stabilizer, is its dual anchor/transporter function (depending on whether Per2 is bound to monoubiquitinated or nonubiquitinated

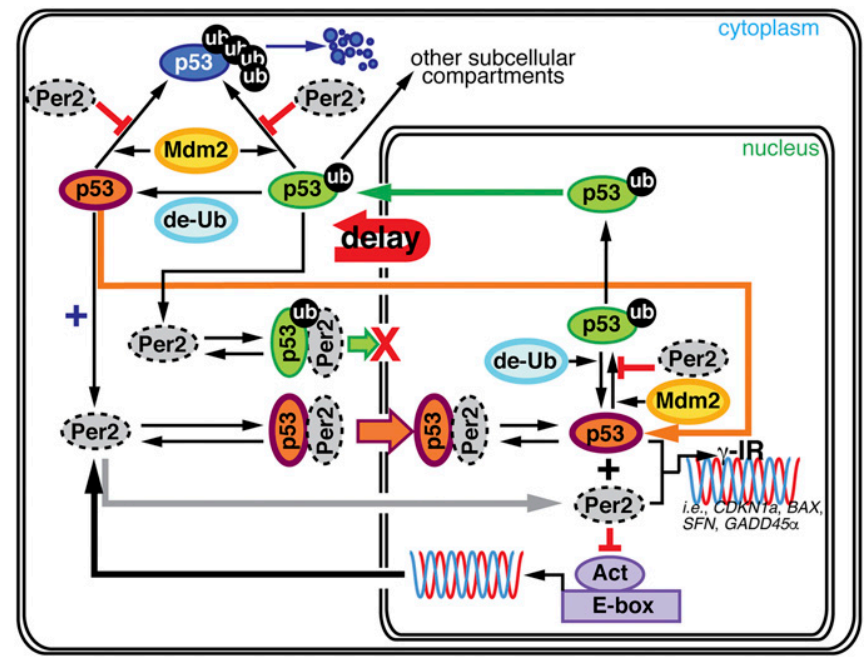

Fig. 6. A unifying model for Per2-mediated regulation of $\mathrm{p} 53$ translocation and signaling. The schematic representation summarized in this section incorporates our previous and newly described findings. First, we focused on the Per2:p53 interaction as a mode of controlling the endogenous levels of p53 under physiological conditions. We proposed this to be a strategy to generate a stable pool of p53 that preconditions the cell for a rapid response to a stress situation (ref. 8; upper left corner of the model). Second, we demonstrated that Per2:p53 interaction is functionally relevant for modulating a p53-mediated transcriptional response in cells exposed to genotoxic stimuli (ref. 9; lower right corner). Last, we show that both processes are related and mediated by events that control the translocation and availability of p53 via Per2 in each subcellular compartment over the course of the circadian cycle (this publication). 
p53) in the Per2:p53 complex, which takes place in a dynamic fashion throughout the day (Fig. 6). We first described the interplay between Per2, p53, and Mdm2, and how Per2:p53 interaction modulates the endogenous levels of $\mathrm{p} 53$ in unstressed conditions, thereby generating a readily available pool of p53 amenable for triggering a rapid response to, for example, genotoxic stress (ref. 8; Fig. 6, upper left corner). Then, we placed Per2 as a direct regulator of 553 expression and transcriptional activity under stress conditions, thus proving that Per2:p53 interaction could be functionally relevant in multiple scenarios (refs. 8 and 9; Fig. 6, lower right corner). Our findings bring both pieces of the Per2 and p53 story together by unveiling the role that Per2-mediated translocation of p53 plays in generating a time-dependent subcellular distribution of the p53's pool (Fig. 6). In a larger context, and considering the functional significance of p53 in normal cell growth and carcinogenesis, understanding the underlying molecular mechanism by which circadian factors regulate p53 accumulation could help identify windows of opportunities for effective chronopharmaceutical treatments.

Last, whereas the focus of our work is in understanding how the circadian clock drives the circadian rhythms of p53 via Per2, it is worth mentioning that other p53 oscillations, with a period of $\sim 5 \mathrm{~h}$, exist and are generated in response to specific stimuli. For example, in MCF7 cells, DNA damage triggers p53 pulses that are primarily driven by those occurring in upstream kinases (e.g., ATM and ChK) and are sustained as long as the DNA damage persists (33). The existence of p53 pulses impacts the expression of different subsets of p53 target genes and, consequently, influences the fate of the cell (34). Interestingly, even unstressed proliferating cells exhibit spontaneous pulses of p53 that are believed to be triggered by intrinsic

1. Mohawk JA, Green CB, Takahashi JS (2012) Central and peripheral circadian clocks in mammals. Annu Rev Neurosci 35:445-462.

2. Takahashi JS, Hong HK, Ko CH, McDearmon EL (2008) The genetics of mammalian circadian order and disorder: Implications for physiology and disease. Nat Rev Genet 9(10):764-775.

3. Rana S, Mahmood S (2010) Circadian rhythm and its role in malignancy. J Circadian Rhythms 8:3.

4. Albrecht U, Bordon A, Schmutz I, Ripperger J (2007) The multiple facets of Per2. Cold Spring Harb Symp Quant Biol 72:95-104.

5. Gery S, Koeffler HP (2010) Circadian rhythms and cancer. Cell Cycle 9(6):1097-1103.

6. Antoch MP, Toshkov I, Kuropatwinski KK, Jackson M (2013) Deficiency in PER protein has no effect on the rate of spontaneous and radiation-induced carcinogenesis. Cell Cycle 12(23):3673-3680.

7. Sancar A, et al. (2015) Circadian clock, cancer, and chemotherapy. Biochemistry 54(2): 110-123.

8. Gotoh T, et al. (2014) The circadian factor Period 2 modulates p53 stability and transcriptional activity in unstressed cells. Mol Biol Cell 25(19):3081-3093.

9. Gotoh T, Vila-Caballer M, Liu J, Schiffhauer S, Finkielstein CV (2015) Association of the circadian factor Period 2 to $p 53$ influences p53's function in DNA-damage signaling. Mol Biol Cell 26(2):359-372.

10. Fu L, Pelicano H, Liu J, Huang P, Lee C (2002) The circadian gene Period2 plays an important role in tumor suppression and DNA damage response in vivo. Cell 111(1):41-50.

11. Sun $\mathrm{CM}$, et al. (2010) Per2 inhibits k562 leukemia cell growth in vitro and in vivo through cell cycle arrest and apoptosis induction. Pathol Oncol Res 16(3):403-411.

12. Hua $\mathrm{H}$, et al. (2007) Inhibition of tumorigenesis by intratumoral delivery of the circadian gene mPer2 in C57BL/6 mice. Cancer Gene Ther 14(9):815-818.

13. Lück S, Thurley K, Thaben PF, Westermark PO (2014) Rhythmic degradation explains and unifies circadian transcriptome and proteome data. Cell Rep 9(2):741-751.

14. Mazan-Mamczarz K, et al. (2003) RNA-binding protein HuR enhances p53 translation in response to ultraviolet light irradiation. Proc Natl Acad Sci USA 100(14):8354-8359.

15. Siepka SM, et al. (2007) Circadian mutant Overtime reveals F-box protein FBXL3 regulation of cryptochrome and period gene expression. Cell 129(5):1011-1023.

16. Hughes ME, Hogenesch JB, Kornacker K (2010) JTK CYCLE: An efficient nonparametric algorithm for detecting rhythmic components in genome-scale data sets. J Biol Rhythms 25(5):372-380.

17. Tamanini F, Yagita K, Okamura H, van der Horst GT (2005) Nucleocytoplasmic shuttling of clock proteins. Methods Enzymol 393:418-435.

18. Marchenko ND, et al. (2010) Stress-mediated nuclear stabilization of p53 is regulated by ubiquitination and importin-alpha3 binding. Cell Death Differ 17(2):255-267.

19. Middeler G, et al. (1997) The tumor suppressor p53 is subject to both nuclear import and export, and both are fast, energy-dependent and lectin-inhibited. Oncogene 14(12):1407-1417

20. Walton KM, et al. (2009) Selective inhibition of casein kinase 1 epsilon minimally alters circadian clock period. J Pharmacol Exp Ther 330(2):430-439.

21. Vanselow K, Kramer A (2007) Role of phosphorylation in the mammalian circadian clock. Cold Spring Harb Symp Quant Biol 72:167-176. and transient DNA damage that occurs during cell cycle transitions (35). Consequently, and in future studies, it would be of interest to investigate the existing interplay between stress-induced pulses of p53 (36) and the circadian rhythms oscillations of p53 (our work) as both the circadian and cell cycle oscillators are synchronized $(37,38)$.

\section{Materials and Methods}

Subcellular fractionation was performed essentially as described (39). Nuclear and cytoplasmic fractions from synchronized HCT116 cells were analyzed for expression of endogenous proteins by immunoblotting following standard procedures (8). When indicated, immunoprecipitation of endogenous Per2 and p53 from nuclei and cytoplasmic fractions were from $8 \times 10^{6}$ and $2 \times 10^{6}$ cells, respectively. To measure protein half-life, fractions were obtained from HCT116 cells collected after $\mathrm{CHX}(100 \mu \mathrm{g} / \mathrm{mL})$ addition $(t=0)$. Endogenous protein levels were determined by immunoblotting and quantitated using the ImageJ software, version 1.45 [NIH software package (40)] or Image Lab Software followed by detection using Gel Doc XR+ System (Bio-Rad). For mathematical modeling, we extended a previous model of the intracellular mammalian circadian clock $(24,25)$ to describe interactions between p53 and Per2 (Fig. $2 A$ and D). Refer to SI Materials and Methods and Table S4 for technical details and Tables S2 and S3 for a list of variables and parameters used in modeling, respectively.

ACKNOWLEDGMENTS. We thank Dr. D. Capelluto for critical reading of the manuscript and Dr. J. Webster for comments and manuscript editing. This work was supported by National Science Foundation Molecular and Cellular Biosciences Division Grant MCB-1517298 and Fralin Life Science Institute (to C.V.F.), and Korea Advanced Institute of Science and Technology Research Allowance Grant G04150020, National Research Foundation of Korea Grant N01160447, and T. J. Park Science Fellowship of POSCO (to J.K.K.).

22. Clodong S, et al. (2007) Functioning and robustness of a bacterial circadian clock. Mol Syst Biol 3:90.

23. Locke JC, Westermark PO, Kramer A, Herzel H (2008) Global parameter search reveals design principles of the mammalian circadian clock. BMC Syst Biol 2:22.

24. Kim JK, Forger DB (2012) A mechanism for robust circadian timekeeping via stoichiometric balance. Mol Syst Biol 8:630.

25. Kim JK (2016) Protein sequestration versus Hill-type repression in circadian clock models. IET Syst Biol 10(4):125-135.

26. Kruse JP, Gu W (2009) Modes of p53 regulation. Cell 137(4):609-622.

27. O'Brate A, Giannakakou P (2003) The importance of p53 location: Nuclear or cytoplasmic zip code? Drug Resist Updat 6(6):313-322.

28. Belle A, Tanay A, Bitincka L, Shamir R, O'Shea EK (2006) Quantification of protein half-lives in the budding yeast proteome. Proc Natl Acad Sci USA 103(35):13004-13009.

29. Yoo SH, et al. (2005) A noncanonical E-box enhancer drives mouse Period2 circadian oscillations in vivo. Proc Natl Acad Sci USA 102(7):2608-2613.

30. Li M, et al. (2003) Mono- versus polyubiquitination: Differential control of $\mathrm{p} 53$ fate by Mdm2. Science 302(5652):1972-1975.

31. Hardin PE, Panda S (2013) Circadian timekeeping and output mechanisms in animals. Curr Opin Neurobiol 23(5):724-731.

32. Kim JK, Forger DB (2012) On the existence and uniqueness of biological clock models matching experimental data. SIAM J Appl Math 72(6):1842-1855.

33. Batchelor E, Mock CS, Bhan I, Loewer A, Lahav G (2008) Recurrent initiation: A mechanism for triggering p53 pulses in response to DNA damage. Mol Cell 30(3): 277-289.

34. Purvis JE, et al. (2012) p53 dynamics control cell fate. Science 336(6087):1440-1444

35. Loewer A, Batchelor E, Gaglia G, Lahav G (2010) Basal dynamics of p53 reveal transcriptionally attenuated pulses in cycling cells. Cell 142(1):89-100.

36. Kim JK, Jackson TL (2013) Mechanisms that enhance sustainability of p53 pulses. PLoS One 8(6):e65242.

37. Bieler J, et al. (2014) Robust synchronization of coupled circadian and cell cycle oscillators in single mammalian cells. Mol Syst Biol 10:739.

38. Feillet C, et al. (2014) Phase locking and multiple oscillating attractors for the coupled mammalian clock and cell cycle. Proc Natl Acad Sci USA 111(27):9828-9833.

39. Schreiber E, Matthias P, Müller MM, Schaffner W (1989) Rapid detection of octamer binding proteins with "mini-extracts", prepared from a small number of cells. Nucleic Acids Res 17(15):6419.

40. Schneider CA, Rasband WS, Eliceiri KW (2012) NIH Image to ImageJ: 25 years of image analysis. Nat Methods 9(7):671-675.

41. Balsalobre A, et al. (2000) Resetting of circadian time in peripheral tissues by glucocorticoid signaling. Science 289(5488):2344-2347.

42. Yang $X$, et al. (2009) $\beta$-catenin induces $\beta$-TrCP-mediated PER2 degradation altering circadian clock gene expression in intestinal mucosa of ApcMin/+ mice. J Biochem 145(3):289-297.

43. Gonzalez OR, Küper C, Jung K, Naval PC, Jr, Mendoza E (2007) Parameter estimation using Simulated Annealing for S-system models of biochemical networks. Bioinformatics 23(4): $480-486$ 